\title{
COMPETITIVE GOMPERTZ MODEL OF TWO SPECIES
}

\author{
LI-CHANG HUNG
}

Abstract. In this paper, a competitive Gompertz model of two species is proposed. Furthermore, under a certain condition, the existence of monotone traveling wave solutions of this model is shown by the method of super- and subsolutions, which is developed in $[6,15,16]$.

Mathematics subject classification (2010): 34C12, 35C07, 35K57, 92D25.

Keywords and phrases: competitive Gompertz model, Lotka-Volterra system, monotone traveling wave solutions, method of super- and subsolutions.

\section{REFERENCES}

[1] D. G. Aronson, H. F. Weinberger, Multidimensional nonlinear diffusion arising in population genetics, Adv. in Math., 30 (1978), 33-76.

[2] R. D. Benguria, M. C. Depassier, Variational characterization of the speed of propagation of fronts for the nonlinear diffusion equation, Comm. Math. Phys., 175 (1996), 221-227.

[3] J. CAnosA, On a nonlinear diffusion equation describing population growth, IBM J. Res. Develop., 17 (1973), 307-313.

[4] N. FEI, J. CARR, Existence of travelling waves with their minimal speed for a diffusing Lotka-Volterra system, Nonlinear Anal. Real World Appl., 4 (2003), 503-524.

[5] K. P. HAdeler, F. Rothe, Travelling fronts in nonlinear diffusion equations, J. Math. Biol., 2 (1975), 251-263.

[6] X. Hou, A. W. LEUnG, Traveling wave solutions for a competitive reaction-diffusion system and their asymptotics, Nonlinear Anal. Real World Appl., 9 (2008), 2196-2213.

[7] L.-C. HUNG, Exact traveling wave solutions for diffusive Lotka-Volterra systems of two competing species, Jpn. J. Ind. Appl. Math., 29 (2012), 237-251.

[8] Y. KAN-ON, Parameter dependence of propagation speed of travelling waves for competition-diffusion equations, SIAM J. Math. Anal., 26 (1995), 340-363.

[9] - Existence of standing waves for competition-diffusion equations, Japan J. Indust. Appl. Math., 13 (1996), 117-133.

[10] - Fisher wave fronts for the Lotka-Volterra competition model with diffusion, Nonlinear Anal., 28 (1997), 145-164.

[11] - Travelling waves for a Lotka-Volterra competition model with diffusion [translation of Sügaku 49 (1997), no. 4, 379-392; MR1614330 (2000b:92020)], Sugaku Expositions, 13 (2000), 39-53.

[12] J. I. KANEL, On the wave front solution of a competition-diffusion system in population dynamics, Nonlinear Anal., 65 (2006), 301-320.

[13] J. I. KANEL, L. ZHOU, Existence of wave front solutions and estimates of wave speed for a competition-diffusion system, Nonlinear Anal., 27 (1996), 579-587.

[14] A. Kolmogorov, I. Petrovsky, N. Piskunov, Etude de lé de la diffusion avec croissance de la quantite de matiere et son applicationa un probleme biologique, Moscow Univ. Math. Bull, 1 (1937), $1-25$.

[15] A. W. Leung, X. Hou, W. Feng, Traveling wave solutions for Lotka-Volterra system re-visited, Discrete Contin. Dyn. Syst. Ser. B, 15 (2011), 171-196.

[16] A. W. LeUnG, X. Hou, Y. LI, Exclusive traveling waves for competitive reaction-diffusion systems and their stabilities, J. Math. Anal. Appl., 338 (2008), 902-924. 
[17] M. Lucia, C. B. Muratov, M. Novaga, Linear vs. nonlinear selection for the propagation speed of the solutions of scalar reaction-diffusion equations invading an unstable equilibrium, Comm. Pure Appl. Math., 57 (2004), 616-636.

[18] M. Rodrigo, M. Mimura, Exact solutions of a competition-diffusion system, Hiroshima Math. J., 30 (2000), 257-270.

[19] M. Rodrigo, M. Mimura, Exact solutions of reaction-diffusion systems and nonlinear wave equations, Japan J. Indust. Appl. Math., 18 (2001), 657-696.

[20] M. TANG, P. FIFE, Propagating fronts for competing species equations with diffusion, Archive for Rational Mechanics and Analysis, 73 (1980), 69-77.

[21] A. I. VOlpert, V. A. Volpert, V. A. Volpert, Traveling wave solutions of parabolic systems, vol. 140 of Translations of Mathematical Monographs, American Mathematical Society, Providence, RI, 1994. Translated from the Russian manuscript by James F. Heyda.

[22] Y. YU, W. WANG, Z. LU, Global stability of Gompertz model of three competing populations, J. Math. Anal. Appl., 334 (2007), 333-348. 